\title{
Assessing performance upon abrupt awakening from naps during quasi-continuous operations
}

\author{
DAVID F. DINGES, MARTIN T. ORNE, and EMILY CAROTA ORNE \\ The Institute of Pennsylvania Hospital and University of Pennsylvania, Philadelphia, Pennsylvania
}

\begin{abstract}
Quasi-continuous work settings often involve sleep loss and requirements to perform at unpredictable times. Napping may alleviate some of the sleep-loss problems, but it increases the risk that the person will have difficulty functioning upon abrupt awakening. This paper describes an experimental approach, techniques, and analyses for investigating performance upon abrupt awakening from 2-h naps placed near either the circadian peak $(P)$ or trough $(T)$ in body temperature and preceded by $6,18,30,42$, or $54 \mathrm{~h}$ of sleep deprivation. Five groups of healthy young adults performed quasi-continuously for $54 \mathrm{~h}$ and were permitted a 2 -h nap at one of five times. Reaction time (RT) to answer a phone terminating the nap, subjective estimates, and performance of a brief, challenging cognitive task were related to nap-sleep parameters of each group. Sleep deprivation increased the amount of deep sleep in the naps, and this was associated with greater postnap cognitive performance decrements; subjective estimates were unaffected, and RT performance was related simply to stage of sleep prior to awakening. Circadian placement of the naps also modulated the postnap cognitive decrement: $T$ naps produced greater cognitive decrements than $P$ naps, even when the latter involved more prior sleep loss. These findings have both practical and theoretical significance for evaluating the awakening process, and would not have been possible without the approach, techniques, and procedures described.
\end{abstract}

There is a fundamental dilemma faced in planning sleep logistics for quasi-continuous work situations, such as those often encountered in the military. The person may have to perform at full capacity, at a moment's notice, at unpredictable times, over a period of many days, weeks, or months. If the individual is kept awake throughout the period, the inevitable effects of sleep deprivation take their toll, performance deteriorates, and involuntary sleep onsets become commonplace. If, on the other hand, the person sleeps, performance upon abrupt awakening from sleep may be severely compromised.

Paradoxically, people actually function less well immediately upon awakening from sleep than they do prior to going to sleep (Kleitman, 1963), although the detrimental effect does not usually last beyond 15 to $30 \mathrm{~min}$ after subjects awaken. Decreased performance upon sudden awakening from sleep has been shown to occur for a broad range of tasks, including simple reaction time (RT) (Dinges, Orne, Evans, \& Orne, 1981; Okuma, Nakamura, Hayashi, \& Fujimori, 1966; Rosa, Bonnet, \& Warm, 1983; Webb \& Agnew, 1964; Wilkinson \&

This research was supported in part by Office of Naval Research Contract No. N00014-80-C-0380 to the first author and in part by a grant from the Institute for Experimental Psychiatry. We gratefully acknowledge the many contributions of Stephen $R$. Fairbrother to all aspects of this research, and thank Wayne G. Whitehouse, John W. Powell, Mary F. Auxier, David A. Soskis, Gordon Hammer, Christine M. Dinges, Christopher Auxier, Ann L. Maliniak, Nancy K. Bauer, Wayne H. Rambo, Barbara R. Barras, and Mae C. Weglarski for their assistance in data collection and analysis and for their helpful suggestions. Reprint requests should be sent to David F. Dinges, Unit for Experimental Psychiatry, 111 North 49th Street, Philadelphia, PA 19139-2798.
Stretton, 1971; Williams, Morlock, \& Morlock, 1966), complex RT (Feltin \& Broughton, 1968; Goodenough, Lewis, Shapiro, Jaret, \& Sleser, 1965; Scott, 1969; Seminara \& Shavelson, 1969), grip strength (Jeanneret \& Webb, 1963; Tebbs \& Foulkes, 1966), steadiness and coordination (Wilkinson \& Stretton, 1971), visualperceptual tasks (Lavie \& Giora, 1973; Scott, 1969; Scott \& Snyder, 1968), memory tasks (Akerstedt \& Gillberg, 1979; Bonnet, 1983; Grosvenor \& Lack, 1984; Stones, 1977), time estimates (Carlson, Feinberg, \& Goodenough, 1978), complex-behavior simulation tasks (Hartman \& Langdon, 1965; Hartman, Langdon, \& McKenzie, 1965; Langdon \& Hartman, 1961; Seminara \& Shavelson, 1969), and a number of cognitive tasks, such as mental arithmetic, cancellation, and clock reversal (Dinges et al., 1981; Fort \& Mills, 1972; Pritchett, 1964/1969; Scott, 1969; Tebbs, 1972; Wilkinson \& Stretton, 1971).

Not only is the immediate postsleep performance decrement ubiquitous, but it also is increased by the depth of sleep. This is evidenced in three ways: (1) Typically, awakenings from non-REM (NREM) sleep stages, especially slow-wave sleep (SWS), yield the greatest decrements; (2) awakenings during the first few hours of nocturnal sleep usually show the most severe decrements; and (3) sleep following prolonged wakefulness yields decrements greater than sleep prior to deprivation. This latter point is particularly germane to the applied problem of sleep logistics in quasi-continuous work settings. Fort and Mills (1972) and Rosa et al. (1983) have found that cognitive and sensory-motor performances of subjects awakening from recovery sleep following sleep loss were below the performance levels of subjects who remained 
awake. That is, performance upon awakening from $2 \mathrm{~h}$ of sleep was worse than the diminished performance capacity of subjects who had continued to remain awake. From a sleep-logistics perspective, such data suggest that it might be better to keep certain people awake continuously than to permit limited sleep (such as 1- or 2-h naps) and thereby risk a transient period of very poor performance immediately after they awaken.

Although the existence of an immediate postsleep performance decrement is widely known, no study has examined the phenomenon in the context of quasi-continuous work demands, for which limited sleep opportunities or naps are thought to be one way of ameliorating the effects of sleep deprivation (Angiboust \& Gouars, 1972; Dinges, Orne, Orne, \& Evans, 1980; Lubin, Hord, Tracy, \& Johnson, 1976; Naitoh, 1981). Furthermore, from a theoretical perspective, work on the phenomenon generally has been based on the assumption that the stage of sleep immediately prior to awakening is the only sleepprocess parameter relevant to the effect; researchers largely have ignored sleep parameters (such as the amount of REM sleep or SWS) other than the stage from which awakening was accomplished. In a preliminary study of performance of subjects awakening from 1-h afternoon naps, however, Dinges et al. (1981) showed that this assumption is valid for immediate postsleep RT performance but that it does not apply to cognitive-task performance upon awakening.

The purpose of this paper is to present an approach for evaluating the immediate postsleep performance decrement and its relationship to sleep infrastructure by using a quasi-continuous work paradigm and a nap study design that permit assessment of both circadian and sleeploss effects on nap-sleep infrastructure and the performance decrement. Procedures are described that permit immediate postsleep assessments without the delays commonly required by a test environment (i.e., lighted sleep room, subjects sitting up, special apparatus). The report shows how sleep loss and circadian factors alter nap-sleep structure, how this alteration in turn affects different kinds of performance immediately upon awakening, and how this relates to planning sleep logistics.

\section{METHOD}

The study reported here is part of a larger investigation of napping in anticipation of, during, and following sleep loss due to quasi-continuous work demands. Napping not only is of applied interest in quasi-continuous settings, but it also provides a theoretically important way of studying immediate postsleep performance decrements without confounding time (circadian variation) and sleep structure (stage changes). In studies of performance of subjects awakening from longer periods of sleep $(4,6$, or $8 \mathrm{~h}$ ), changes in sleep structure are confounded with circadian changes in a manner that obscures the major source of variance contributing to the specific decrement. This is perhaps the primary reason why stage of sleep at awakening is often the only sleep parameter assessed. On the other hand, studies of 1- or 2-h naps at different phases of the circadian cycle permit specific aspects of sleep to be related to performance upon awakening, and by varying prior sleep loss, the structure and depth of sleep can be related to the degree of decrement upon awakening.

A limitation to this kind of nap paradigm is that it tends to restrict the complexity of sleep and therefore may not reveal all factors that contribute to a performance decrement. This limitation does not appear to be a significant problem, however, because the data to date suggest that the worst performance decrements occur when subjects awaken in the first few hours of nocturnal sleep, that is, approximately the length of a 2-h nap. The first few hours of sleep tend to comprise NREM stages, particularly SWS, which was found in an earlier study of 1-h naps to be associated with the severity of the cognitive performance decrement upon awakening (Dinges et al., 1981).

Because neither the structure of sleep nor the severity of the performance decrement upon awakening are under the direct control of the experimenter, the approach used is necessarily quasi-experimental. The circadian and sleepdeprivation manipulations are intended to alter sleep structure, and this change in sleep structure is hypothesized to increase the severity of the cognitive performance decrement upon awakening. The approach is, therefore, also correlational. Sleep deprivation should increase the amount of SWS in a 2-h nap, and this should be associated with a more severe immediate postnap cognitive performance decrement. The relationship is not expected to be linear over time, however, because circadian variation should, to some degree, modulate sleep structure and depth of sleep, as well as the ability to maintain wakefulness, thereby also affecting the extent of the performance decrement upon awakening.

\section{Design}

The present investigation of immediate postsleep performance is only one aspect of a larger study of napping during sustained operations. The design of the overall study necessarily involves measurement components that are not relevant to this report. For example, performance testing on a variety of tasks every 3 or $4 \mathrm{~h}$ throughout $54 \mathrm{~h}$ of sustained operations was an integral part of the overall study, but the bulk of these performance tasks have no bearing on either the assessment or the analysis of performance upon abrupt awakening. Details of their administration and results are not reported here.

The dependent variables of interest were the subjects' cognitive and sensory-motor performance upon abrupt termination of a 2-h napping period and the sleepinfrastructure characteristics of the nap. The independent variables were the amount of sleep loss prior to the nap and the phase of the circadian cycle in which the nap was taken. The null hypothesis stated that sleep deprivation prior to naps and circadian-phase placement of naps will not affect either the sleep infrastructure of the naps or immediate postnap performance. A corollary of this is that 
there will be no relationship between any aspect of napsleep infrastructure and the extent of the postnap performance decline.

The design considered most appropriate to address this issue involved five independent groups of subjects; each group represented a different treatment condition. Figure 1 shows the placement of the 2-h nap opportunity for each of the five groups during the $54 \mathrm{~h}$ of sustained wakefulness with quasi-continuous performance demands. As the graph suggests, the groups differed from each other in the amount of sleep loss prior to the nap and in the time of day (i.e., phase of the endogenous circadian temperature cycle) when the nap was permitted. For example, the first group, designated P-6, received a 2-h nap opportunity before any significant sleep loss; their nap occurred the first afternoon, near the circadian peak $(P)$ in oral temperature, after only $6 \mathrm{~h}$ of wakefulness since nocturnal sleep offset. In comparison, the T-42 group received a nap opportunity after $42 \mathrm{~h}$ of sustained wakefulness and near the circadian trough $(\mathrm{T})$ in core temperature.

Although this approach necessarily confounds the phase of circadian cycle with the amount of prior sleep loss, there is no way around this problem because both of these factors are temporal dimensions that covary with each other. By considering data from the $P$ and $T$ groups separately, one can address the sleep-deprivation factor without a circadian confound. However, there are applied reasons for wanting to compare $\mathbf{P}$ and $\mathrm{T}$ groups. Naitoh (1981) presented data showing that the recuperative power of a 2-h nap following sleep deprivation depends upon the phase of the circadian cycle in which the nap is taken. A $T$ nap after $42 \mathrm{~h}$ of sleep loss was not nearly as beneficial as a $P$ nap after $54 \mathrm{~h}$ of sleep loss. Although these comparisons pose a theoretical problem, they provide potentially crucial information for assessing the cost of napping at different times during sustained operations.

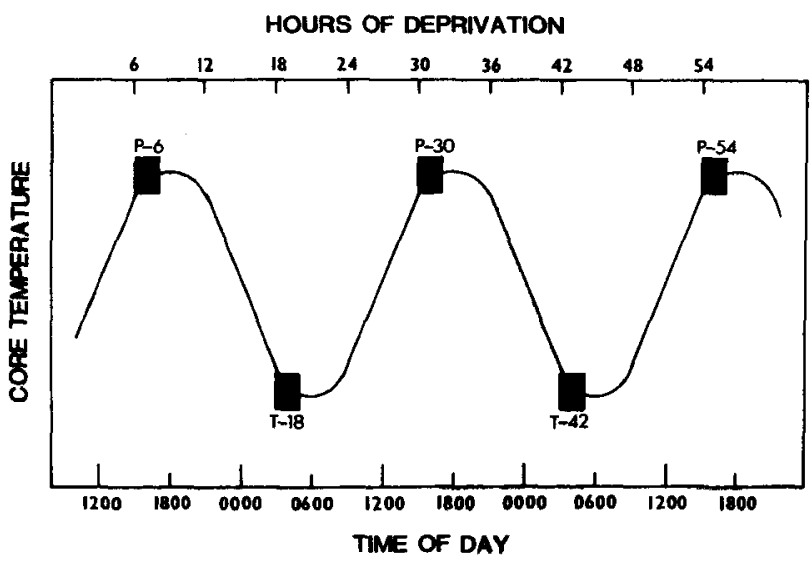

Figure 1. Placement of a 2-h nap for each of five experimental groups during $54 \mathrm{~h}$ of quasi-continuous performance demands. Groups are identified by the time at which nops occurred in the idealized circadian cycle $(P=$ near the peak, $T=$ near the trough) and by the hours of sleep deprivation prior to the naps $(6,18,30,42$, $54 \mathrm{~h})$.

\section{Subjects}

A total of 35 healthy young adults ( 20 males and 15 females), ranging in age from 18 to 30 years, participated in the study. They were assigned randomly in same-sex pairs to one of the five experimental groups, with the restriction that both sexes had to be represented in each experimental group. The male:female ratio was $5: 2$ in the P-6 group, 4:2 in the T-18 group, 2:4 in the P-30 group, 4:4 in the T-42 group, and 5:3 in the P-54 group. (Additional subjects are currently being run to make comparisons between genders within each condition; there are too few subjects to make this possible at this time.) One male subject in the P- 6 condition was found to have consumed excessive amounts of alcohol the night before the 54-h deprivation period began, and his data were therefore excluded. The subjects were run in same-sex pairs through the 54-h deprivation period, with the exception of one group of three females run together. The subjects in each pairing had not met prior to deprivation, and both underwent the same experimental treatment condition.

Because the design required the subjects to nap at different times of day following varying amounts of sleep deprivation, we sought to broadly ensure that all volunteers had had some experience with napping and sleep loss and that they did not have abnormally phase-shifted sleep/wake cycles. Moreover, because there are widespread intersubject differences in response to sleep deprivation that have never been adequately understood, we sought to minimize the contribution of factors that might increase these differences. Aside from pseudorandom assignment to treatment conditions, all subjects were screened prior to the experiment with a number of questionnaires to determine whether sleep/wake patterns were within reasonable limits and to reduce gross heterogeneity of sleep patterns.

First, subjects had to qualify as healthy replacement nappers on the Survey of Subjective Sleep Patterns (Dinges et al., 1980); that is, they had to be free of sleep onset, maintenance, or offset problems, they had to nap at least once a week when they felt tired as the result of shortened nocturnal sleep, and they had to find naps to be generally satisfying. Healthy replacement nappers consistently make up about 33\% of all college adults (Dinges et al., 1980).

Second, the subjects had to have experienced at least 1 night (circa $20 \mathrm{~h}$ ) of sleep loss since the age of 18 years. This was assessed by a Sleep/Wakefulness Survey, which is a questionnaire that we developed to permit subjective self-report of prior experiences and reactions to sleep deprivation. Over $75 \%$ of the population surveyed met the criterion of having experienced at least 1 night of voluntary sleep loss. Third, the subjects -were assessed for nonextreme scores on the Morningness-Eveningness questionnaire (Horne \& Ostberg, 1976).

A senior investigator confirmed qualifying reponses to each of the screening questionnaires by conducting two laboratory interviews with prospective subjects during the baseline portion of the study. Although all subjects who participated met each of the criteria, there was no attempt 
made to match subjects in each treatment condition according to the details of these criteria, such as number of naps per week, longest period of prior sleep deprivation, or morningness-eveningness score.

The subjects who agreed to participate in the study were reimbursed a total of $\$ 163.90$ ( $\$ 150.00$ for their time, $\$ 10.00$ for completing a sleep diary, and $\$ 3.90$ for transportation to and from the laboratory). All subjects who participated successfully completed the sleepdeprivation experiment, although the subjects were informed ahead of time that they could withdraw at any time without jeopardy. None of the subjects demonstrated any major medical or psychological problems during the course of the experiment. All subjects were extensively debriefed at the end of the deprivation period, and followup contact was maintained for 2 weeks after the study.

\section{Procedures}

Subjects who met the qualifying questionnaire criteria were scheduled for three experimental sessions. The first two sessions were each $5 \mathrm{~h}$ in length and involved: completion of additional sleep questionnaires and personality inventories; interviews about sleep/wake patterns and experiences with sleep loss; self-report measures of mood; oral temperature readings; and baseline practice on various performance tasks, including 10-min simple visual RT (Glenville, Broughton, Wing, \& Wilkinson, 1978), 10-min simple auditory RT (Lisper \& Kjellberg, 1972), 3-min descending subtraction task (DST) (Evans \& Orne, 1975), digit-span (DS) task, 20-min Probe-Recognition Memory Task (adapted from Elkin \& Murray, 1974, and Pozella, 1974), Memory and Search Task (Folkard, Knauth, Monk, \& Rutenfranz, 1976), and the Stroop Color-Naming Task (Golden, 1978).

The subjects were tested individually during the two baseline sessions. The first session occurred 7 to 14 days prior to the second session. During this time, the subjects were asked to complete a sleep diary (Dinges et al., 1980) each morning after they awakened. This diary provided self-report information on each of the subjects' sleep/wake cycles prior to the sleep-deprivation session. The subjects were told to maintain their typical sleep patterns between the first two sessions.

The third experimental session took place 1 to 4 days after the second session. The subjects were instructed to get a normal night's sleep on the 2 nights prior to the third session and to be certain that they did not sleep-deprive themselves more than usual on these nights. If they were not already awake, they were awakened by a phone call at $0900 \mathrm{~h}$ the morning of the third session and were scheduled to report to the laboratory as soon as possible after $1200 \mathrm{~h}$ that day. The actual range in arrival time was 1200 to $1600 \mathrm{~h}$.

Testing on predetermined subsets of performance tasks began as soon as subjects arrived at the laboratory and continued throughout the next $54 \mathrm{~h}$. Each test bout included both visual and auditory RT tasks, as well as selected cognitive tasks. Performance bouts averaged $45 \mathrm{~min}$ in duration, with a range of $40-60 \mathrm{~min}$. The bouts occurred, on average, every $3 \mathrm{~h}$, with a range of 1 to $4 \mathrm{~h}$. The beginning and end of each bout were marked by the subjects' oral temperatures being taken while they completed the Stanford Sleepiness Scale (Hoddes, Zarcone, Smythe, Phillips, \& Dement, 1973), the Profile of Mood States (McNair, Lorr, \& Droppleman, 1971), and analogue ratings of sleepiness (Evans \& Orne, 1975), fatigue, and tension (Dinges et al. 1980).

Although participating in pairs, the subjects were tested individually on performance tasks. First, one was tested, and then the other-the order was constant throughout the deprivation period. Between performance bouts, the subjects were free to engage in a variety of activities, including reading, conversation, board games, studying, darts, and cards. Monitors stayed with them at all times between test bouts to ensure that they remained awake, and a senior investigator administered the performance tests without the aid of a computer (see Ryman, Naitoh, \& Englund, 1984, for a description of a new computerbased system for task administration).

During deprivation, meals were scheduled at the same time of day for all groups, and included breakfast, lunch, supper, and a late night snack. Food was also available between meals if subjects desired it. Although all groups received basically the same food, no attempts other than these were made to control dietary variables (e.g., amount eaten, caloric or carbohydrate intake, etc.). Smoking was permitted, but only three subjects (each in a different group) smoked during or outside the experiment. No caffeinated beverages were permitted, and no subject showed signs of caffeine withdrawal.

The deprivation environment was temperature controlled and devoid of explicit time-of-day cues. There were no clocks present; windows were occluded; radio, television, and phone calls were not permitted; and staff refrained from discussing time. This condition was intended only to prevent the subjects from being biased in their mood responses by knowing the exact hour of the day. There was no expectation that they would lose track of time during what was a relatively brief deprivation period.

Depending upon treatment condition, subjects were permitted naps sometime during the deprivation period. As with test bouts, the paired subjects were staggered by about $1 \mathrm{~h}$ in terms of when the nap actually occurred. First, one subject was prepared and permitted to sleep; then, the second subject was prepared and permitted to sleep in a separate bedroom. The nap took place on a bed, in a dark, quiet room with an ambient temperature of $70^{\circ} \mathrm{F}$. Subjects slept in regular clothing but were permitted to cover themselves with a sheet and blanket.

Immediately prior to being allowed to nap, after sleeprecording electrodes had been attached and the subjects had lain in the bed in the darkened room, they were required to perform the DST and the DS task over an inter- 
com hookup between the polygraph recording room and the sleep room. Following this, subjects were told that they could go to sleep.

They were awakened from the nap $2 \mathrm{~h}$ later. Awakening was achieved by a telephone's ringing next to the bed. (The sound-attenuated bedrooms prevented the phone from disturbing subjects in the other bedroom.) A light on the phone lit when the phone rang. Subjects were informed prior to the nap that the phone would ring, signaling the end of the nap, and that they should answer the phone as soon as possible. An electronic circuit recorded on the polygraph the lengths of time the bell rang before the subjects broke the circuit by lifting the receiver. These times served as the RT measures immediately upon awakening.

After answering the phone, the subjects were asked two questions and then instructed to hang up the phone, lie back (in the dark), and perform the DST and the DS task. These immediate postnap performance trials, as well as all performance bouts throughout the experiment, were audiotape-recorded to permit careful examination of performance and interactions. As with the RT measure upon awakening, the subjects were told prior to the nap that they would be asked to perform the DST and DS tasks immediately after they had answered the phone. After the DS task, the electrodes were removed and the subjects were taken to the testing room for a performance bout.

Regardless of nap condition, subjects continued in deprivation-with the quasi-continuous testing protocoluntil approximately $1800 \mathrm{~h}$ on the 3rd day. By this time, they had undergone $54 \mathrm{~h}$ of sleep deprivation, with approximately $16 \mathrm{~h}$ of performance testing. The final $2 \mathrm{~h}$ of the deprivation period were devoted to an extensive postexperimental debriefing of subjects by an experimenter who had not been involved with the testing or monitoring of subjects up to that time.

\section{Instructions}

When the experiment was explained to subjects in an effort to solicit participation, no mention was made of the hypotheses concerning the effects of nap sleep as a function of different amounts of prior sleep deprivation and at different phases of the circadian cycle. Rather, the study was explained as an attempt to understand individual differences in response to sleep deprivation; the sleep deprivation and quasi-continuous performance demands were detailed, and the subjects were told that "sometime during the sleep-deprivation period, at least once and possibly twice, you will be permitted to take a nap." It was further explained that this could occur at any time from immediately after they had entered the laboratory at the beginning of deprivation to just before they left at the end of deprivation, and anytime in between. Subjects were told that, when the nap did occur, its duration could be anywhere from $10 \mathrm{~min}$ to $5 \mathrm{~h}$. It was explained that the precise timing of the nap and its duration would not be known to them ahead of time. The purpose of this was to simulate a quasi-continuous work situation, in which sleep opportunities and durations are generally not known ahead of time.

Once in the deprivation session, subjects were not told when a test bout was coming, how long it would last, when it would end, or when another would begin. They were urged to perform at their maximum ability each time they performed the tasks, including immediately after the nap, and were not provided feedback about any of their previous responses. They were told to expect to perform the DST and DS tasks immediately upon awakening from the nap.

\section{Instrumentation}

Physiological measures. Oral temeprature was recorded at the beginning and the end of each performance bout and immediately before and after the nap, by a mercury bulb thermometer. Nap-sleep physiology was recorded on a Grass Model 7 polygraph and was scored (30-sec epochs) according to standardized criteria (Rechtschaffen \& Kales, 1968). Parameters included vertex and occipital electroencephalograms, electrooculograms, electromyograms, and electrocardiograms. Dependent variables consisted of: total sleep time (TST); amount of Stages wake, 1, 2, 3, 4, REM, SWS, and NREM sleep; stage of sleep within seconds of the bell; sleep latency (defined by Stage 1 and Stage 2 onsets); and latency to SWS and REM.

Reaction time. The time it took subjects to answer the telephone that signaled the end of the nap was recorded electronically on the polygraph. This measure had been used in earlier work and had been shown to be related to stage of sleep upon awakening (Dinges et al., 1981). One of the subjects in each pair was exposed to a 72-dB bell (SPL re: $.0002 \mathrm{dyne} / \mathrm{cm}^{2}$ ), whereas the other was exposed to a 93-dB bell. The bell remained on until the subject lifted the receiver. In some cases, especially with severely sleep-deprived subjects who napped and then were exposed to the $72-\mathrm{dB}$ bell, the phone was not picked up after 1 min of ringing; when this occurred, it was then turned on and off every second for $10 \mathrm{sec}$. If the subjects still had not answered it, the experimenter asked the subjects if they were awake, and continued to do so until the subjects had answered.

Subjective estimates. Upon picking up the receiver, subjects were asked, "How long has it been since I spoke to you last?" After answering, subjects were asked to rate their sleepiness on a 10-point analogue scale, on which " 1 is very wide awake, absolutely no desire to sleep, and 10 is very groggy, sleep is imminent."

Cognitive task. The DST was administered immediately before subjects were told to "go to sleep" and immediately after the subjective estimates had been made when the phone was answered. In the past, the DST had tended to show the most dramatic decrements and carryover effects of sleep. The task was specially designed in our laboratory to tax the cognitive functioning of an individual for a relatively brief time. Because it can be carried out by subjects who are lying in bed in the dark, 
without the presence of the experimenter in the room, it allows testing within a few seconds of sleep offset.

The task consists of giving subjects a three-digit number, such as 672 , which they are asked to repeat aloud as the first response. The subjects are then required to mentally subtract the number 9 from 672 and to say the answer (663) aloud. This answer then becomes the new minuend from which 8 must be subtracted. Thus, the subtrahend progressively decreases by 1 until, having reached the value of 2 , it returns to 9 , and the series is continued for $3 \mathrm{~min}$. The instructions emphasize repeatedly that the subjects "should work as fast as possible and keep a steady pace, but try to remain as accurate as possible." A sequence of correct subtractions would be: $672,663,655,648,642,637,633,630,628,619$, $611,604,598$, etc. The subtractions are performed silently, with only the answers being spoken aloud.

The task clearly requires that both the subtrahend and minuend be kept in mind. Because both change after each response, a considerable load is placed on short-term memory. Subjects are encouraged to correct any wrong responses, but, in any case, to go on. Subjects are instructed that if they become lost in a sequence, they are to continue, even if they have to guess. In this experiment, if subjects did not respond on the DST for $30 \mathrm{sec}$, the experimenter then said, "Please continue, guess if you have to," and thereafter pushed the subject every few seconds to respond. (This proved to be necessary only for some DST performances immediately upon subjects' awakening.) Thus, subjects could not score poorly on the task by merely not responding for the bulk of the $3 \mathrm{~min}$ allotted.

Subjects' responses were written down by the experimenter and also recorded on audiotape; the audiotapes were used to fill in any responses missed by the experimenter. (This latter precaution proved critical for evaluating immediate postnap DST performance of subjects who spoke in a soft voice, mumbled, or spoke aloud intrusions of fantasy while doing the task.) The polygraph was kept running while the subjects performed the DST immediately upon awakening from the nap. The task was scored for speed (total number of responses), accuracy (number of errors), and both speed and accuracy (number correct per unit time). The DST has a substantial practice effect over the first nine trials. This was overcome by having subjects perform the task at least a dozen times before even the earliest (P-6) postnap treatment.

\section{RESULTS}

Table 1 presents the means of nap-sleep parameters as well as the means of the immediate postnap behavioral measures for each group. A major between-group effect on nap-sleep parameters was evident on sleep onset, TST, Stage 1 sleep, SWS, SWS latency, and sleep stage at the bell terminating the nap. The difference that accounted for most of the variance was between the non-sleepdeprived P-6 group and all other sleep-deprived groups,
Table 1

Means and $F$ Ratios Between Groups

\begin{tabular}{|c|c|c|c|c|c|c|c|}
\hline Dependent Variable & P-6 & $\mathrm{T}-18$ & P-30 & $\mathrm{T}-42$ & P-54 I & $F(4,29)$ & $\mathbf{p}<$ \\
\hline \multicolumn{8}{|c|}{ Nap-Sleep Measures (in Minutes) } \\
\hline Sleep Onset* & 22.2 & 7.2 & 6.8 & 6.5 & 4.5 & 4.4 & .01 \\
\hline Total Sleep Time & 65.3 & 104.5 & 106.3 & 113.8 & 113.2 & 14.7 & .001 \\
\hline Stage 1 Sleep & 22.9 & 8.3 & 10.4 & 4.7 & 3.3 & 9.4 & .001 \\
\hline Stage 2 Sleep & 34.9 & 44.5 & 30.0 & 37.7 & 26.7 & 1.8 & \\
\hline Stages $3+4$ Sleep (SWS) & 19.5 & 55.7 & 62.2 & 68.9 & 77.5 & 16.3 & .001 \\
\hline Stage REM Sleep & 10.9 & 4.3 & 14.2 & 7.3 & 9.1 & 1.6 & \\
\hline SWS Latency* & 41.2 & 16.5 & 11.7 & 5.1 & 4.3 & 4.3 & .01 \\
\hline REM Latency* & 73.8 & 67.1 & 72.2 & 63.1 & 68.2 & 0.3 & \\
\hline Sleep Stage at Bell & 1.4 & 2.8 & 2.3 & 2.6 & 2.9 & 3.9 & .025 \\
\hline \multicolumn{8}{|c|}{ Postnap Behavioral Measures } \\
\hline 1/RT to Bell & 0.3 & 0.1 & 0.2 & 0.1 & 0.1 & 4.2 & .01 \\
\hline Temporal Estimate (min) & 97.5 & 121.3 & 79.2 & 95.7 & 146.3 & 2.4 & \\
\hline 10-Point Sleepiness Rating & 5.7 & 3.8 & 5.7 & 5.6 & 6.3 & 1.1 & \\
\hline DST\% Correct Prenap & 73.4 & 61.6 & 62.4 & 29.1 & 29.1 & 7.3 & .001 \\
\hline
\end{tabular}

Note-Sleep Stage at Bell is not presented in minutes, but rather, is scored such that each discrete stage is assigned a value (wake $=0$, Stage I $=1$, Stage $R E M=1.5$, Stage $2=2$, Stage $3=3$, Stage $4=4$ ), thereby yielding an "average" Stage at Bell along a discrete continuum from wakefulness to Stage 4 sleep. Postnap behavioral measures were each scored differently. Reaction Time to Bell was scored as the reciprocal of the seconds to answer the phone; Temporal Estimate was scored in minutes as the response to the question How long since I spoke to you last?"; Sleepiness Rating was scored on a 10-point scale, where 1 represented awake; and DST\% Prenap was the immediate postnap DST number correct as a percentage of the immediate prenap DST number correct. $\quad *$ Measured from Stage 2 sleep onset.

especially the T-42 and P-54 groups. Not surprisingly, sleep-deprived groups averaged faster sleep onset, more TST, less Stage 1, more SWS, a shorter SWS latency, and a deeper stage at the bell.

Post hoc Sheffe' tests (Kirk, 1968) also revealed differences between sleep-deprived groups on certain parameters. The most severely deprived group, P-54, had significantly shorter sleep onset times, more TST, less Stage 1, more SWS, and a shorter SWS latency than the T-18 and P-30 groups. Table 1 indicates that groups tended to cluster into three separate zones on those sleep parameters that yielded significant $F$ ratios; the values for the P- 6 group represent one zone, the values for the T-18 and $\mathrm{P}-30$ groups represent another, and the values for the T-42 and P-54 groups represent the third zone (see TST, Stage 1, SWS, and SWS latency means).

Postnap behavioral measures yielded significant between-group differences for RT to the bell and DST performance decrement, but not for either subjective estimate. [The actual RT measure used in these analyses was the reciprocal of RT (i.e., 1/RT), which is recommended when the treatment means and standard deviations are proportional (see Kirk, 1968, p. 66); with reciprocal transformation, longer $\mathrm{RTs}$ are represented by smaller numbers.] The 1/RT F ratio was accounted for by the difference between the P-6 group and all other groups. This result closely paralleled that found for stage of sleep at bell; the P-6 group averaged a light 1.4 stage and the fastest RT $(1 / \mathrm{RT}=0.3)$, the P-30 group averaged a 2.3 stage and the next fastest RT (0.2), and the T-18, $T-42$, and P-54 groups averaged 2.6 to 2.9 stages and the slowest RTs $(0.1)$. (Stage at bell was calculated by assign- 
ing values to each of the standardized categories; wakefulness $=0$, Stage 1 sleep $=1$, REM sleep $=1.5$, Stage 2 sleep $=2$, Stage 3 sleep $=3$, Stage 4 sleep $=$ 4.) When groups were pooled, the highest correlation between 1/RT and nap sleep occurred, as expected, for stage at bell $[\mathrm{r}(32)=-.60, \mathrm{p}<.05$, when adjusted for the number of correlations conducted; see Larzelere \& Mulaik, 1977]. Deeper stages of sleep, and Stage 4 in particular, yielded substantially slower RTs (i.e., smaller 1/RT values).

Interestingly, the waking bell intensity did not affect RT. Those 17 subjects who were exposed to the $72-\mathrm{dB}$ bell averaged a $2.35(\mathrm{SD}=0.91)$ stage of sleep at the bell and a $0.16(\mathrm{SD}=0.17) \mathrm{RT}$ reciprocal. The $17 \mathrm{ex}-$ posed to the $93-\mathrm{dB}$ bell averaged a $2.56(\mathrm{SD}=0.90)$ stage and a $0.13(\mathrm{SD}=0.09) \mathrm{RT}$ reciprocal. The $\mathrm{t}$ values between groups were below 0.7 in both cases.

Cognitive DST performance immediately after the nap was below that of immediately before the nap for all groups. The percentage of prenap performance presented in Table 1 shows that, like certain nap-sleep parameters, the DST grouped into zones: The P-6 group had the least decrement (down 26\% from prenap), the T-18 and P-30 groups averaged a greater decrement (down $38 \%$ ), and the T-42 and P-54 groups averaged the greatest decrement (down 71\%). Scheffé tests indicated that these differences were significant among the groups in each zone. Correlations between the extent of DST decrement and nap-sleep parameters produced the highest coefficient for amount of SWS $[\mathrm{r}(32)=-.63, \mathrm{p}<.05$, when adjusted for number of correlations conducted]. The more SWS in the nap, the greater was the immediate postnap DST decrement.

\section{DISCUSSION}

The results clearly indicate that the experimental hypotheses were supported. The manipulation of increasing sleep deprivation produced deeper nap sleep, in terms of both amount of SWS and stage at bell, and greater immediate postnap RT and DST performance decrements. This was most dramatic for DST cognitive performance. Consistent with an earlier study of 1-h naps in non-sleepdeprived subjects (Dinges et al., 1981), RT upon awakening was associated with stage of sleep immediately prior to awakening, whereas DST performance was associated with the amount of SWS accumulated in the nap, rather than merely stage of sleep immediately prior to abrupt awakening.

The fact that amount of SWS relates most closely to cognitive decrement upon awakening suggests that something accumulates psychophysiologically during SWS that makes it difficult for subjects to function cognitively when they are aroused within close temporal proximity of the SWS process. The process appears to dissipate gradually or to reverse its detrimental cognitive effect as the amount of time elapsed since SWS increases. This is apparently what many of us refer to as the waking process: Something changes when subjects awaken, and this change permits them to function in an alert manner.

This period of confusion upon awakening has been called sleep inertia (Lubin et al., 1976), sleep drunkenness (Broughton, 1968), and postdormital disorientation (Association of Sleep Disorders Centers, 1979). Certain clinical disorders of arousal, such as sleepwalking and night terrors, also appear to be associated with it, particularly with awakenings from SWS (Broughton, 1968). In our study, one cannot help but be struck by the clinical similarity to some of these SWS arousal disorders, especially the cognitive performance of subjects who were sleep-deprived for 42 to $54 \mathrm{~h}$. In most of these cases, the decrement is characterized by profound disorientation, inability to accomplish correct subtractions, bizarre hypnogogic intrusions that are spoken while the subject gives DST answers, and later amnesia for the events. This is all the more dramatic when one considers that subjects expected to have to perform the task at awakening, they were not asked to perform it until they had motorically responded to a bell and had answered two questions, and the experimenter forced them to interact and provide responses.

Interestingly, circadian-phase placement of the nap did appear, as hypothesized, to modulate the effects of sleep deprivation on nap-sleep and cognitive-task decrement. Naps in the circadian trough produced sleep patterns (stages and amounts) and cognitive decrements nearly identical to naps taken $12 \mathrm{~h}$ later, near the circadian peak. Conversely, peak naps produced considerably less cognitive decrement than did trough naps taken $12 \mathrm{~h}$ later. The best example of this is seen when the P-30 group performance is compared with the $\mathrm{T}-42$ group performance. Despite relatively equivalent nap-sleep means, especially SWS, the T-42 subjects, upon awakening, suffered a cognitive-task performance decrement that was nearly twice that of the P-30 subjects. This result is consistent with the results of Fort and Mills (1972) and Naitoh (1981) in suggesting that circadian influences and sleepdeprivation influences on immediate postsleep performance are additive. Thus, it appears that circadian influences at the trough add considerably to the cognitive performance decrement, whereas at the peak they add little, and perhaps even attenuate, the decrement. The relationship between the amount of sleep deprivation and the extent of the decrement is, therefore, nonlinear due to these interactions.

This finding suggests that, from the standpoint of napping in quasi-continuous work settings, particularly those that may require optimum performance at a moment's notice, 2-h naps in the circadian trough should probably be avoided. This is clearly counterintuitive in that sleep tendency is higher near the circadian trough than near the peak. In addition, naps should probably be taken before sleep loss accumulates beyond $36 \mathrm{~h}$, because more severe cognitive performance decrements can be expected upon awakening from naps taken after this time.

The results of performance between naps in this and further studies will be required before a firm consensus 
can be reached concerning the utility of naps to facilitate functioning during quasi-continuous work demands. However, these results of performance upon abrupt awakening suggest that this facet of functioning should be considered when sleep logistics are planned for personnel who may have to perform complex tasks with minimal warning.

The nap paradigm, study design, tasks, procedures, and quasi-experimental approach used in this investigation of performance upon abrupt awakening have provided novel theoretical and practical information. It is noteworthy that the approach that we employed involved (1) varying both the amount of sleep loss and the circadian phase of the 2-h naps, (2) assessing all sleep parameters, (3) relating these parameters to performance on a specially developed cognitive task, and (4) administering this task immediately upon subjects' awakening. Had we not used sleep loss and circadian phase to vary the amount of SWS, or had we focused only on the stage of sleep prior to subjects' awakening, or had we assessed task performance many minutes after their awakening (as is typically done), we would not have been able to document the relationship between cognitive decrement and SWS. Instead, we would have had to attempt to explain the between-group differences in cognitive decrements according to minimal differences in sleep stage at awakening. This would have made little sense and been of no value in terms of assessing the effects of varying amounts of sleep loss and circadian-phase placement of naps. Although much remains to be learned about performance upon awakening, including the duration of the decrement and factors that might attenuate it, we hope that future investigators will consider using the approach, techniques, and analyses described here.

\section{REFERENCES}

Akerstedt, T., \&illberg, M. (1979). Effects of sleep deprivation on memory and sleep latencies in connection with repeated awakenings from sleep. Psychophysiology, 16, 49-52.

ANGiboust, R., \& GouARS, M. (1972). Tentative d'evaluation de l'efficacite operationelle du personnel de l'aeronautique militaire au cours de veille's nocturnes. In W. P. Colquhoun (Ed.), Aspects of human efficiency (pp. 151-170). London: English University Press. Association of Sleep Disorders Centers. (1979). Diagnostic classification of sleep and arousal disorders (1st ed.) [Special issue]. Sleep, 2, 1-137.

BONNET, M. H. (1983). Memory for events occurring during arousal from sleep. Psychophysiology, 20, 81-87.

Broughton, R. J. (1968). Sleep disorders: Disorders of arousal? Science, 159, 1070-1078.

Carlon, V. R., Feinberg, I., \& Goonenough, D. R. (1978). Perception of the duration of sleep intervals as a function of EEG sleep stage. Physiological Psychology, 6, 497-500.

Dinges, D. F., Orne, E. C., Evans, F. J., \& Orne, M. T. (1981). Performance after naps in sleep-conducive and alerting environments. In L. C. Johnson, D. I. Tepas, W. P. Colquhoun, \& M. J. Colligan (Eds.), Biological rhythms, sleep and shifi work: Advances in sleep research (Vol. 7, pp. 539-552). New York: Spectrum.

Dinges, D. F., Orne, M. T., Orne, E. C., \& Evans, F. J. (1980). Voluntary self-control of sleep to facilitate quasi-continuous perfor- mance (Annual Summary Rep. No. 80). Frederick, MD: U.S. Army Medical Research and Development Command. (NTIS No. ADA 102264)

Elkin, A. J., \& Murray, D. J. (1974). The effects of sleep loss on short-term recognition memory. Canadian Journal of Psychology, 28, 192-198.

EVANS, F. J., \& ORNE, M. T. (1975). Recovery from fatigue (Annual Summary Rep. No. 60). Frederick, MD: U.S. Army Medical Research and Development Command. (NTIS No. AD-A100347)

Feltin, M., \& Broughton, R. (1968). Differential effects of arousal from slow wave versus REM sleep. Psychophysiology, 5, 231. (Abstract)

Folkard, S., Knauth, P., Monk, T. H., Rutenfranz, J. (1976). The effect of memory load on the circadian variation in performance efficiency under a rapidly rotating shift system. Ergonomics, 19 479-488.

ForT, A., \& MiLLs, J. N. (1972). Influence of sleep, lack of sleep and circadian rhythm on short psychometric tests. In W. P. Colquhoun (Ed.), Aspects of human efficiency (pp. 115-127). London: English University Press.

Glenville, M., Broughton, R., Wing, A. M., \& Wilkinson, R. T. (1978). Effects of sleep deprivation on short duration performance measures compared to the Wilkinson Auditory Vigilance Task. Sleep, $1,169-176$.

Golden, C. J. (1978). The Stroop Color and Word Test: A manual for clinical and experimental uses. Chicago: Stoelting.

Goodenough, D. R., LeWIS, H. B., Shapiro, A., JARET, L., \& Sleser, I. (1965). Dream report following abrupt awakening from different kinds of sleep. Journal of Personality and Social Psychology, 2, 170-179.

Grosvenor, A., \& LACK, L. C. (1984). The effect of sleep before or after learning on memory. Sleep, 7, 155-167.

HARTMAN, B. O., \& LANGDON, D. E. (1965). A second study on performance upon sudden awakening (Report No. TR-65-61) Brooks Air Force Base, TX: United States Air Force, School of Aerospace Medicine.

Hartman, B. O., Langdon, D. E., \& McKenzie, R. E. (1965). A third study on performance upon sudden awakening (Report No. TR65-63). Brooks Air Force Base, TX: United States Air Force, School of Aerospace Medicine.

Hoddes, E., Zarcone, V., Smythe, H., Phillips, R., \& Dement, W. C. (1973). Quantification of sleepiness: A new approach. Psychophysiology, 10, 431-436.

HORNE, J. A., \& OSTrERG, O. (1976). A self-assessment questionnaire to determine morningness-eveningness in human circadian rhythms. International Journal of Chronobiology, 4, 97-110.

JEANNERET, P. R., \& WEBB, W. B. (1963). Strength of grip on arousal from full night's sleep. Perceptual and Motor Skills, 17, 759-761.

KIRK, R. E. (1968). Experimental design: Procedures for the behavioral sciences. Belmont, CA: Wadsworth.

KLeItman, N. (1963). Sleep and wakefulness. Chicago: University of Chicago Press.

Langdon, D. E., \& HARTMan, B. O. (1961). Performance upon sudden awakening (Report No. 62-17). Brooks Air Force Base, TX: United States Air Force, School of Aerospace Medicine.

LARZELERE, R. E., \& Mulalk, S. A. (1977). Single-sample tests for many correlations. Psychological Bulletin, 84, 557-569.

LAVIE, P., \& GIORA, Z. (1973). Spiral aftereffect durations following awakening from REM and non-REM sleep. Perception \& Psychophysics, 14, 19-20.

LiSPER, H., \& KJELLBERG, A. (1972). Effects of a 24-hour sleep deprivation on rate of decrement in a 10-minute auditory reaction time task. Journal of Experimental Psychology, 96, 287-290.

Lubin, A., Hord, D., Tracy, M. L., \& Johnson, L. C. (1976). Effects of exercise, bedrest and napping on performance decrement during 40 hours. Psychophysiology, 13, 334-339.

MCNAIR, D. M., LORR, M., \& DropPleman, L. F. (1971). EITS manual for the Profile of Mood States. San Diego: Educational and Industrial Test Services.

NaITOH, P. (1981). Circadian cycles and restorative power of naps. 
In L. C. Johnson, D. I. Tepas, W. P. Colquhoun, \& M. J. Colligan (Eds.), Biological rhythms, sleep and shifiwork: Advances in sleep research (Vol. 7, pp. 553-580). New York: Spectrum.

Okuma, T., Nakamura, K., Hayashi, A., \& Fujimori, M. (1966). Psychophysiological study on the depth of sleep in normal human subjects. Electroencephalography and Clinical Neurophysiology, 21, 140-147.

Pozella, D. J. (1974). The effect of sleep deprivation on short-term memory (Tech. Rep. No. 47). Ann Arbor, MI: University of Michigan, Human Performance Center.

Pritchett, T. P. (1969). An investigation of sudden arousal from rest: A study of impaired performance on an addition task (Doctoral dissertation, University of Kentucky, 1964). Dissertation Abstracts International, 30, 2934B. (University Microfilms No. 69-20,443)

ReChTSCHAFFeN, A., \& Kales, A. (Eds.). (1968). A manual of standardized terminology, techniques and scoring system for sleep stages of human subjects (NIH Publication No. 204). Washington, DC: U.S. Government Printing Office.

Rosa, R. R., BonNET, M. H., \& WARM, J. S. (1983). Recovery of performance during sleep following sleep deprivation. Psychophysiology, 20, 152-159.

Ryman, D. H., Naitoh, P., \& Englund, C. E. (1984). Minicomputeradministered tasks in the study of effects of sustained work on human performance. Behavior Research Methods, Instruments, \& Computers, 16, 256-261.
ScotT, J. (1969). Performance after abrupt arousal from sleep: Comparison of a simple motor, a visual-perceptual, and a cognitive task. Proceedings of the 77th Annual Convention of the American Psychological Association, 4, 225-226.

SCOTT, J., \& SNYDER, F. (1968). "Critical reactivity" (Pieron) after abrupt awakenings in relation to EEG stages of sleep. Psychophysiology, 4, 370.

Seminara, J. L., \& Shavelson, R. J. (1969). Effectiveness of space crew performance subsequent to sudden sleep arousal. Aerospace Medicine, 40, 723-727.

STONES, M. J. (1977). Memory performance after arousal from different sleep stages. British Journal of Psychology, 68, 177-181.

Tebis, R. B. (1972). Post-awakening visualization performance as a function of anxiety level, REM or NREM sleep, and time of night. (Report No. TR-72-005). United States Air Force Academy, CO: United States Air Force, Seiler Research Laboratory.

TEBBS, R. B., \& FoulKEs, D. (1966). Strength of grip following different stages of sleep. Perceptual and Motor Skills, 23, 827-834.

WEBB, W. B., AGNEW, H., JR. (1964). Reaction time and serial response efficiency on arousal from sleep. Perceptual and Motor Skills, 18, 783-784.

Wilkinson, R. T., \& STRETTON, M. (1971). Performance after awakening at different times of night. Psychonomic Science, 23, 283-285.

Williams, H. L., Morlock, H. C., JR., \& Morlock, J. V. (1966). Instrumental behavior during sleep. Psychophysiology, 2, 208-216. 